CVIA

REVIEW ARTICLE

pISSN 2508-707X / elSSN 2508-7088 https://doi.org/10.22468/cvia.2018.00129 CVIA 2018;2(3):110-122

\title{
Updates on Transcatheter Aortic Valve Replacement and the Role of Multi-Detector Computed Tomography: What a Radiologist Should Know
}

\author{
Yuan-Heng Mo1,2, Yen-Shu Kuo ${ }^{1,3}$, \\ Yung-Cheng Wang ${ }^{1}$, Ming-Ting $\mathrm{Wu}^{4,5}$ \\ 'Department of Radiology, Cathay General Hospital, Taipei, Taiwan \\ ${ }^{2}$ Department of Radiology, National Taiwan University Hospital and National Taiwan University \\ College of Medicine, Taipei, Taiwan \\ ${ }^{3}$ Graduate Institute of Biomedical Electronics and Bioinformatics, National Taiwan University, \\ Taipei, Taiwan \\ ${ }^{4}$ Department of Radiology, Kaohsiung Veterans General Hospital, Kaohsiung, Taiwan \\ ${ }^{5}$ Faculty of Medicine, School of Medicine, National Yang Ming University, Taipei, Taiwan
}

Received: May 18, 2018

Revised: June 22, 2018

Accepted: June 29, 2018

Corresponding author

Ming-Ting Wu, MD

Department of Radiology,

Kaohsiung Veterans General Hospital, Kaohsiung, Taiwan;

Faculty of Medicine, School of Medicine, National Yang-Ming University,

Taipei, Taiwan:

Institute of Clinical Medicine, National Yang-Ming University, No. 386, Ta-Chung 1st Road,

Kaohsiung 81362, Taiwan

Tel: 886-7-3422121

Fax: 886-73468301

E-mail:wu.mingting@gmail.com
The indications of the transcatheter aortic valve replacement (TAVR) procedure have been widely expanded and the number of cases has significantly increased in the past 15 years due to continued evolution of prosthetic devices, increased experience of cardiologists, and advancements in the cardiovascular imaging field. In this review article, we summarize updates on TAVR guidelines, state-of-the-art prosthetic devices, recommended pre- and post-procedural multi-detector computed tomography (MDCT) protocols, required imaging parameters and measurements, and post-procedural complications. We also address issues concerning the Asian population and patients with bicuspid aortic valve and emphasize some of the challenges ahead. MDCT is the recommended modality of choice for pre-procedural planning for TAVR and current guidelines state that MDCT should be performed by well-trained personnel and interpreted and analyzed by experienced radiologists who not only have a sound understanding of TAVR with respect to its indications, prosthetic devices, procedure, and complications, but also work in close collaboration with the Heart Team.

Key words Transcatheter aortic valve replacement - Multidetector computed tomography . Aortic valve stenosis · Aortic dissection.

\section{INTRODUCTION}

Aortic valve stenosis (AS) is the most common progressive valvular disease in adults and shows an increased incidence in the elderly, making it a disease burden that cannot be overlooked in aging/aged societies. Aortic valve replacement is usually required for patients with severe AS. The mortality rate is dismal and increases to $50 \%$ within 2 years as patients with symptomatic AS progress to heart failure [1,2]. Before the ad-

(c) This is an Open Access article distributed under the terms of the Creative Commons Attribution Non-Commercial License (https://creativecommons.org/licenses/bync/4.0) which permits unrestricted non-commercial use, distribution, and reproduction in any medium, provided the original work is properly cited. vent of transcatheter aortic valve replacement (TAVR), surgical aortic valve replacement (SAVR) remained the standard treatment for patients with severe AS, reporting both low mortality and good clinical outcomes $[3,4]$. However, the treatment choice has tilted towards the much less invasive TAVR procedure since it was first introduced 15 years ago. To date, several clinical trials have demonstrated either superiority or non-inferiority of TAVR to SAVR, especially in inoperable or high surgical risk patients [5-7]. More recent studies have suggested the feasibility of expanding the indication of TAVR to intermediate or even low surgical risk populations [8-11]. As a result of this growing evidence the case number of TAVR procedures has been 
rapidly growing worldwide and has surpassed that of SAVR in recent years [12].

\section{NEW GUIDELINES FOR TAVR}

General rules should be applied when considering the choice of treatment for AS according to the latest 2017 the American Heart Association/the American College of Cardiology (AHA/ ACA) and the European Society of Cardiology/the European Association for Cardio-Thoracic Surgery (ESC/EACTS) guidelines, as summarized in Table 1 [13-16]. The guidelines recommend surgical risk stratification in terms of morbidity and mortality using the Society of Thoracic Surgeons (STS) score and the European System for Cardiac Operative Risk Evaluation (EuroSCORE) II for adults undergoing cardiac surgery [17-19]. These scores are derived from the demographic and clinical vari- ables of the patient and the website-based calculators can be accessed as follows: STS score, http://riskcalc.sts.org/stswebriskcalc/\#/calculate; EuroSCORE II, http://www.euroscore.org/calc. $\mathrm{html}$. A low surgical risk is defined as an STS/EuroSCORE II $<4 \%$ in the absence of other established risk factors including frailty, porcelain aorta, and sequelae of chest radiation. For AS patients who are not stratified as low risk, TAVR is favored over SAVR in the absence of severe comorbidities that suggest SAVR is unlikely to improve the quality of life or survival. In such cases the patients should be carefully and individually evaluated by the Heart Team based on clinical, anatomical, technical, cardiac, and extracardiac characteristics. It should be noted that current evidence on TAVR is limited to patients older than 75 years, and that the long-term durability of TAVR prosthetic devices has not been established to date [12].

Table 1. Factors to be considered by the Heart Team for decision making between SAVR and TAVR, based on the recommendations in the American Heart Association/the American College of Cardiology (AHA/ACA) and the European Society of Cardiology/the European Association for Cardio-Thoracic Surgery (ESC/EACTS) 2017 guidelines on the management of valvular heart disease

\begin{tabular}{|c|c|c|}
\hline & Favors TAVR & Favors SAVR \\
\hline \multicolumn{3}{|l|}{ Clinical characteristics } \\
\hline STS/EuroSCORE II <4\% & & + \\
\hline STS/EuroSCORE II $\geq 4 \%$ & + & \\
\hline Presence of severe comorbidity & + & \\
\hline Age $<75$ years & & + \\
\hline Age $\geq 75$ years & + & \\
\hline Previous cardiac surgery & + & \\
\hline Frailty & + & \\
\hline Restricted mobility and conditions that may affect the rehabilitation process after the procedure & + & \\
\hline Suspicion of endocarditis & & + \\
\hline \multicolumn{3}{|l|}{ Anatomical and technical aspects } \\
\hline Favorable access for transfemoral TAVR & + & \\
\hline Unfavorable access (any) for TAVR & & + \\
\hline Sequela of chest radiation & + & \\
\hline Porcelain aorta & + & \\
\hline Presence of intact coronary bypass grafts at risk when sternotomy is performed & + & \\
\hline Expected patient-prosthesis mismatch & + & \\
\hline Severe chest deformation or scoliosis & + & \\
\hline Short distance between coronary ostia and aortic valve annulus & & + \\
\hline Size of aortic valve annulus out of range for TAVR & & + \\
\hline Aortic root morphology unfavorable for TAVR & & + \\
\hline Valve morphology unfavorable for TAVR (bicuspid, degree of calcification, calcification pattern) & & + \\
\hline Presence of thrombi in aorta or LV & & + \\
\hline \multicolumn{3}{|l|}{ Cardiac conditions in addition to aortic stenosis that require consideration for concomitant intervention } \\
\hline Severe $\mathrm{CAD}$ requiring revascularization by $\mathrm{CABG}$ & & + \\
\hline Severe primary mitral valve disease, which could be treated surgically & & + \\
\hline Severe tricuspid valve disease & & + \\
\hline Aneurysm of the ascending aorta & & + \\
\hline Septal hypertrophy requiring myectomy & & + \\
\hline
\end{tabular}

CABG: coronary artery bypass grafting, CAD: coronary artery disease, EuroSCORE: European System for Cardiac Operative Risk Evaluation, LV: left ventricle, SAVR: surgical aortic valve replacement, STS: Society of Thoracic Surgeons, TAVR: transcatheter aortic valve replacement 


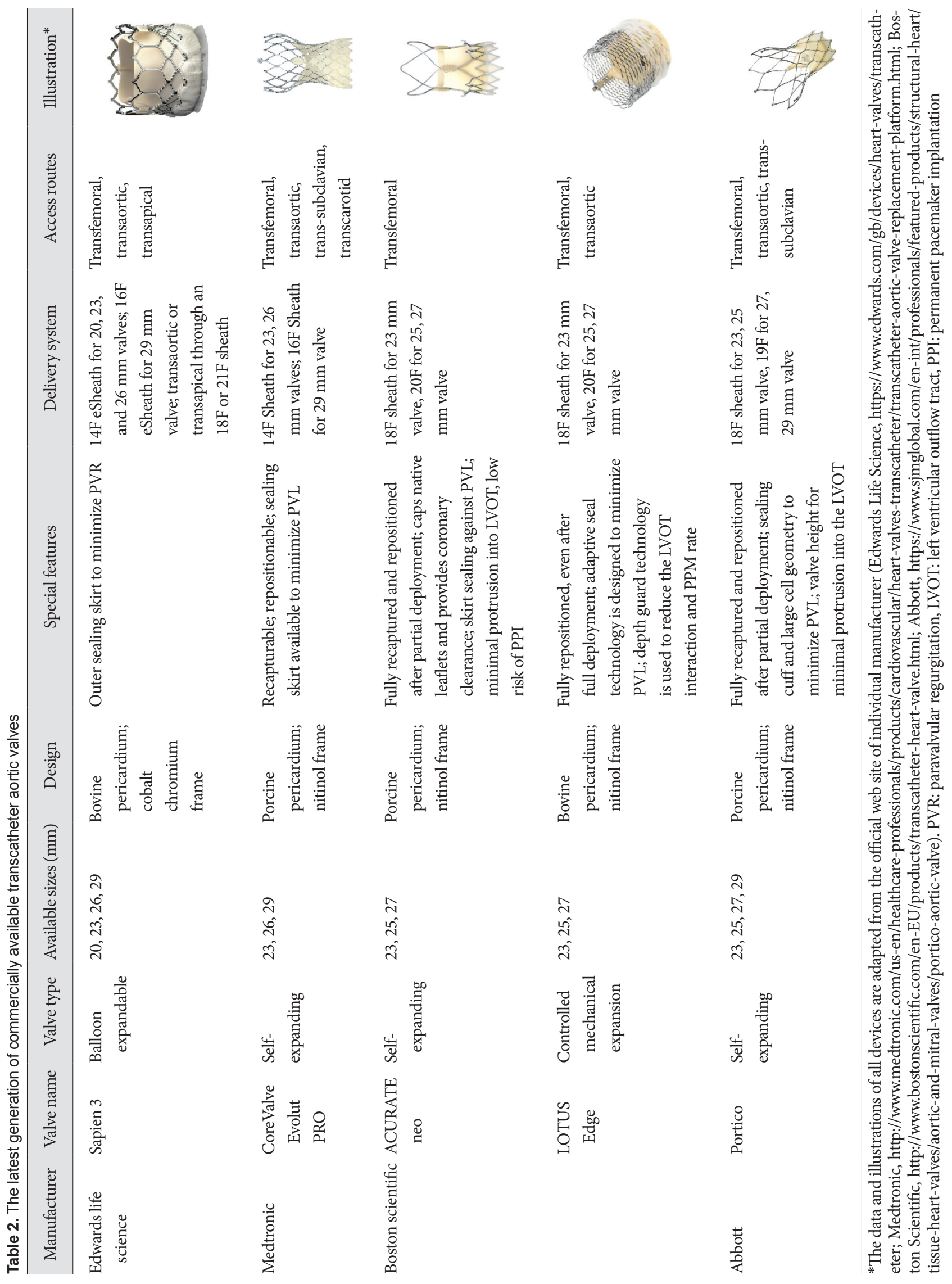




\section{TYPES OF PROSTHESIS}

Two major types of transcatheter aortic valve prostheses have been widely studied in randomized clinical trials-the balloon-expandable and self-expanding prostheses [20] distributed by Edwards life science and Medtronic respectively. The latest commercially available prosthetic devices are summarized in Table 2 [20,21]. Compared with the balloon-expandable prosthesis, advantages of the self-expanding prosthesis include its repositionability, no need for rapid ventricular pacing in order to alleviate hemodynamic disturbance, and a lower risk of annular rupture. However, complications such as paravalvular regurgitation (PVR) and permanent pacemaker implantation (PPI) are more commonly seen with the self-expanding prosthesis. Newer devices have been reported to have a lower complication rate but more solid evidence from large-scale randomized direct comparison studies is still lacking [22,23].

\section{PRE-TAVR EVALUATION}

The 2017 AHA/ACC and ESC/EACTS guidelines state that multi-detector computed tomography (MDCT) is the preferred imaging modality for pre-procedural evaluation of patients undergoing TAVR. The imaging assessment should encompass the following: the size and shape of the aortic root and the aortic valve annulus, the distance from aortic annulus to coronary ostia, the distribution of calcifications and the number of aortic valve cusps, and evaluation of the possible peripheral access routes including the minimal/maximal luminal diameters, the atherosclerotic plaque burden, and the degree of tortuosity of the vessels, as well as presence of aneurysms and/or thrombi [13]. Cardiovascular abnormalities such as valvular disease (e.g., bicuspid aortic valve), cardiac mass, septal hypertrophy, intracardiac thrombus (Fig. 1), or aortic aneurysm should also be documented.

\section{MDCT PROTOCOLS}

The Society of Cardiac Computed Tomography (SCCT) expert consensus document on MDCT imaging for pre-procedural planning for TAVR suggests the use of an at least 64-slice CT scanner equipped with a wide detector ("the wider the better") for faster acquisition time and contrast volume reduction [24]. The recommended MDCT protocols summarized in Table 3 have been modified from technical documents from Medtronic and the SCCT expert consensus. In general, for evaluation of aortic root, most centers use an electrocardiography (ECG)gated contrast enhanced systolic phase MDCT scanning technique; for evaluation of the peripheral access routes, non ECGgated MDCT angiography with high pitch is usually adopted to reduce the radiation dose.

As with any other cardiovascular imaging, TAVR MDCT is not without limitations. First, administration of heart rate lowering agents (e.g., $\beta$-blockers) is not always possible because the typical cohort consists of mostly elderly individuals with a high incidence of comorbidities such as atrial fibrillation, and this can result in image degradation. Contrast-induced nephropathy could also be a concern in an elderly patient group [25]. The radiation dose of TAVR MDCT is relatively high but can be reasonably reduced with automated tube current modulation and iterative image reconstruction. Image acquisition using a dual-source MDCT with prospective ECG gating, a high pitch of 3.4, and a low tube voltage of $100 \mathrm{kVp}$ could reduce the radiation dose to less than $5 \mathrm{mSv}$ and decrease the required amount of contrast agent at a lower injection rate, while still allowing for adequate vascular enhancement without sacrificing the image quality [26]. Such an approach could be beneficial for patients with severe renal insufficiency [27,28].

In terms of image evaluation and CT measurements, a sequential double-oblique reconstruction that best reveals the anatomy
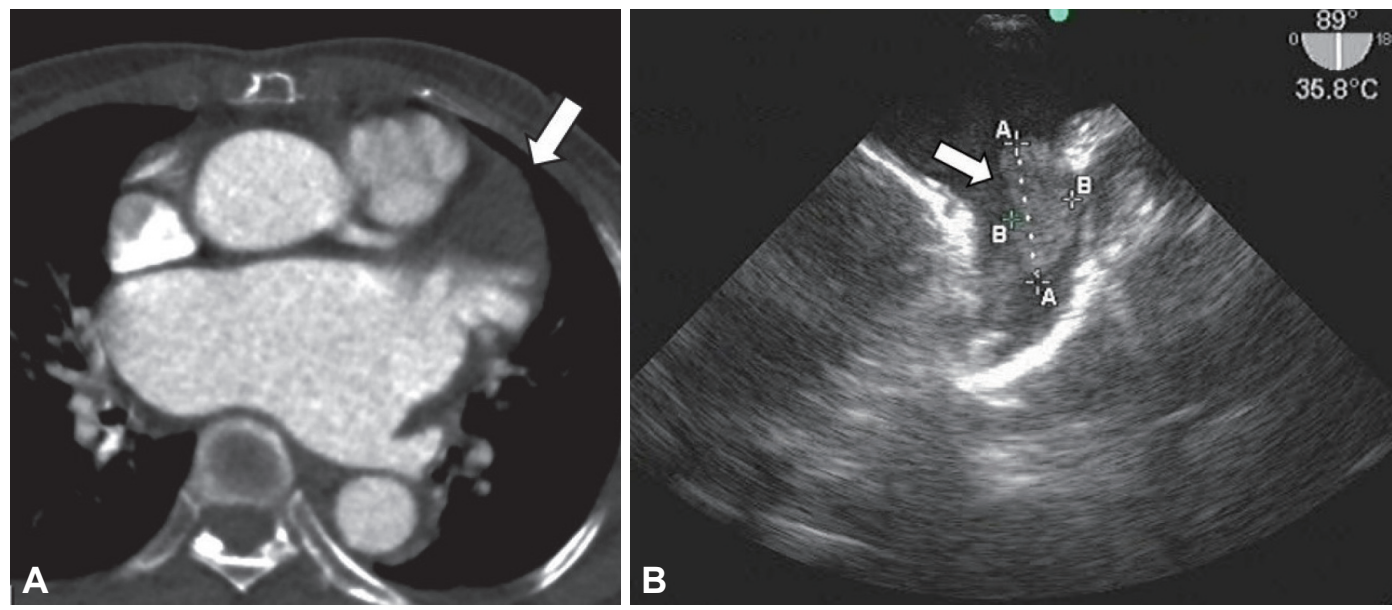

Fig. 1. A 71-year-old female with severe aortic valve stenosis and atrial fibrillation. Pre-TAVR multi-detector computed tomography (A) and transesophageal echocardiography $(B)$ revealed a large thrombus (white arrow) in the left atrial appendage. Surgical aortic valve replacement was performed instead of TAVR for thrombus removal. TAVR: transcatheter aortic valve replacement. 
Updates on Transcatheter Aortic Valve Replacement and the Role of Multi-Detector Computed Tomography

Table 3. Pre-procedural transcatheter aortic valve replacement multi-detector computed tomography protocol recommendations

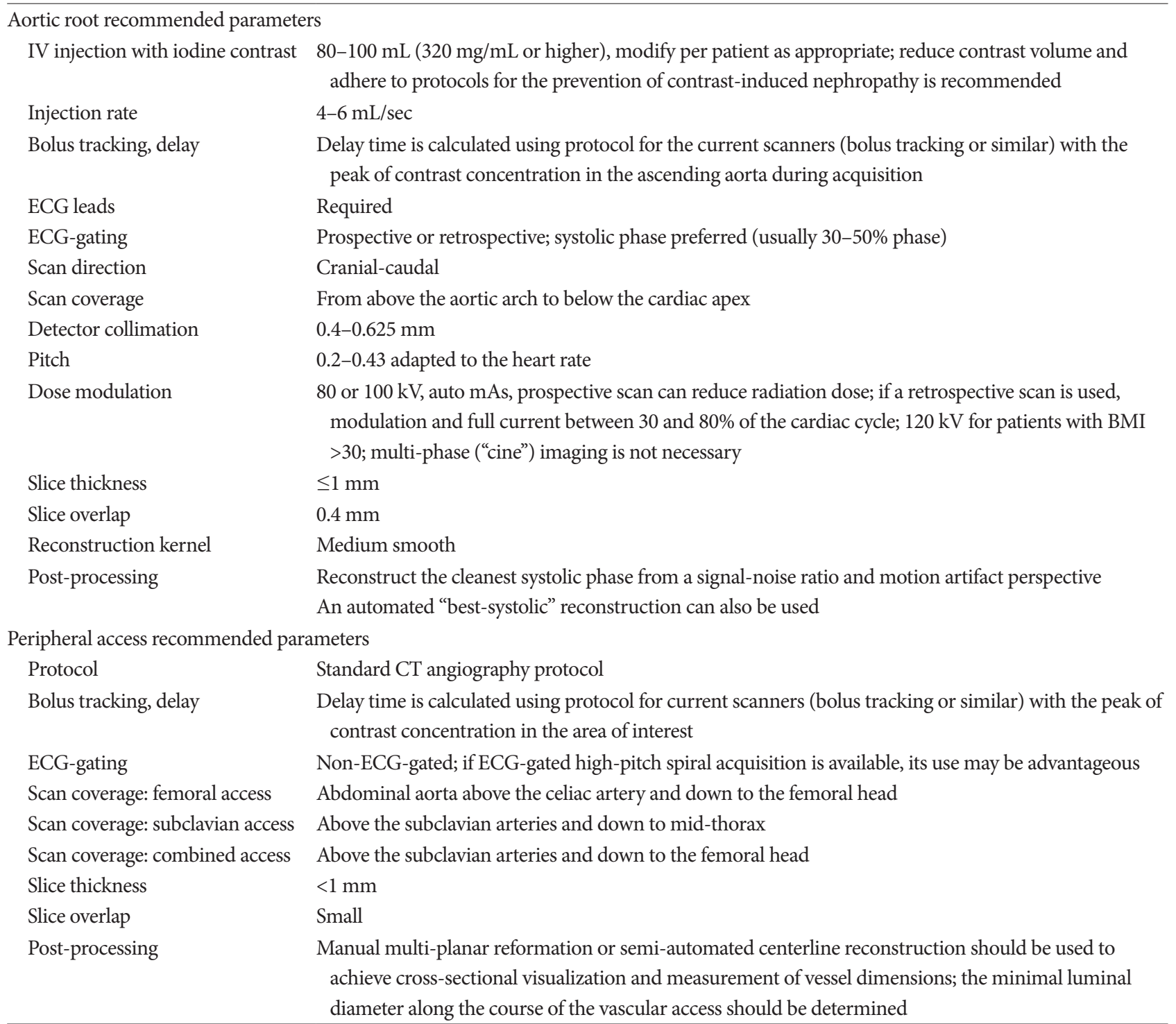

ECG: electrocardiography, BMI: body mass index

of the aortic valve and annulus is most commonly used. Postprocessing methods for assessing the aortic root and the ascending aorta have been described in detail by Achenbach et al. [24] and Leipsic et al. [29]. It is pivotal to accurately establish the size of the aortic annulus in order to optimize prosthetic device selection (Fig. 2). The required parameters and measurements for pre-procedural TAVR MDCT are summarized in Table $4[24,25,30]$. The routine MDCT measurements are illustrated in Fig. 3.

\section{ACCESS ROUTES}

The transfemoral approach is the preferred route of access for TAVR whenever feasible. Alternative routes include transaortic, trans-subclavian, and transapical access. Although less commonly used, transcarotid, transcaval, and antegrade aortic routes are also possible [31]. The choice of access route depends on the prosthetic device selected, the physical properties of the corresponding delivery system, and the adequacy of the vascular routes being investigated. Prominent atherosclerotic wall calcification (specifically circumferential), too small a diameter of the native vessel (smaller than the outer diameter of the delivery sheath to be used), and marked tortuosity of the iliac arteries in the case of transfemoral approach have been identified as risk factors for procedural complications. When two or more of these features are present in the iliofemoral arteries, an alternative transapical, trans-subclavian, or transaortic approach could be considered $[25,32]$. 

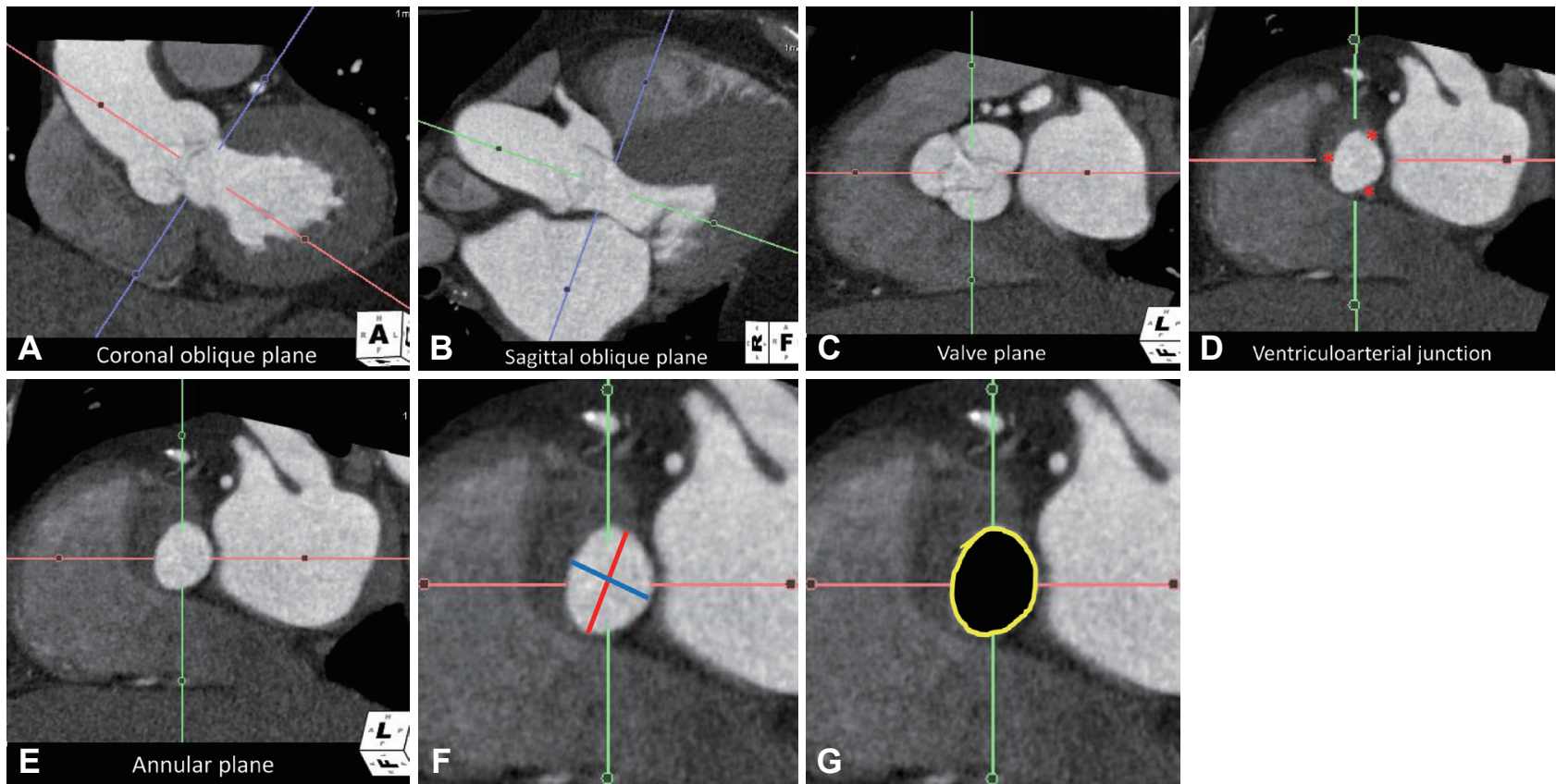

Fig. 2. Steps in aortic annulus measurement. 1) Use of the double-oblique reconstruction plane method to correctly reformat the image along the valve plane (A, B, C). 2) Identification of the lowest points of all three aortic valve leaflets (red *)-the ventriculoarterial junction on one transverse image (D). 3) The bottom of the aortic root is formed by the virtual ring, the aortic annulus-the annular plane (E). The annular plane image can be used to measure the true long-axis (red line in F) and short-axis (blue line in F) annular diameters, the annular area (black area in G), and the annular perimeter (yellow circle in G).

\section{TAVR PROCEDURE}

TAVR is usually performed under general anesthesia and transesophageal echocardiography (TEE) guidance in a hybrid operating room [23]. After the access route has been secured, balloon aortic valvuloplasty is first performed to dilate the stenotic native aortic valve, allowing for a better passage of the transcatheter prosthetic device to its final position. Next, the unexpanded prosthetic device is introduced and its position checked; if the position is optimal, deployment of the prosthetic device is initiated while monitoring in a plane perpendicular to the aortic annulus. For the balloon-expandable device, balloon expansion is conducted during rapid ventricular pacing in order to minimize cardiac output and prevent device migration during deployment. In contrast, the self-expanding device does not require ventricular pacing during deployment because of its self-expanding frame, which conforms to the native aortic annulus [25]. Both improper positioning of the prosthetic device during deployment and undersizing of the annulus, which results in insufficient grip on the aortic wall, may lead to device migration (Fig. 4) [33].

\section{POST-TAVR COMPLICATIONS}

\section{Paravalvular regurgitation}

Preventing or minimizing the amount of PVR post-TAVR is essential in order to improve long-term clinical outcomes [34].
The following factors have been shown to correlate with the occurrence and severity of PVR: 1) valve undersizing, 2) incorrect positioning of the prosthetic valve, and 3) incomplete contact of the prosthetic device to the native aortic annulus due to annular/valve calcifications [25]. Some of the newer prosthetic devices with design modifications such as sealing skirts have yielded a lower PVR rate [21].

\section{Conduction defects requiring permanent pacemaker implantation}

Device expansion within the annulus may cause extrinsic compression to the atrioventricular node and bundle branch fibers due to their close anatomical proximity. Compared with their balloon-expandable counterparts, self-expanding prostheses have a higher rate of conduction disorder. This is partly attributable to their increased length, which plunges deeper into the left ventricular outflow tract (LVOT) and possibly induces a conduction deficit [23]. The number of cases requiring postTAVR PPI has been reduced with newer prosthetic devices [21].

\section{Cerebrovascular events}

The risk of 30-day stroke after TAVR is similar across different prosthetic devices despite varied treatment approaches (e.g., 2.4\% for CoreValve vs. 3.0\% for Edwards Valve in one multicenter registry) [35]. With technological improvements in prosthetic devices, refined patient selection, and increased operator experience, a decline in post-TAVR stroke incidence 
Updates on Transcatheter Aortic Valve Replacement and the Role of Multi-Detector Computed Tomography

Table 4. Required multi-detector computed tomography parameters and measurements in pre-procedural transcatheter aortic valve replacement evaluation

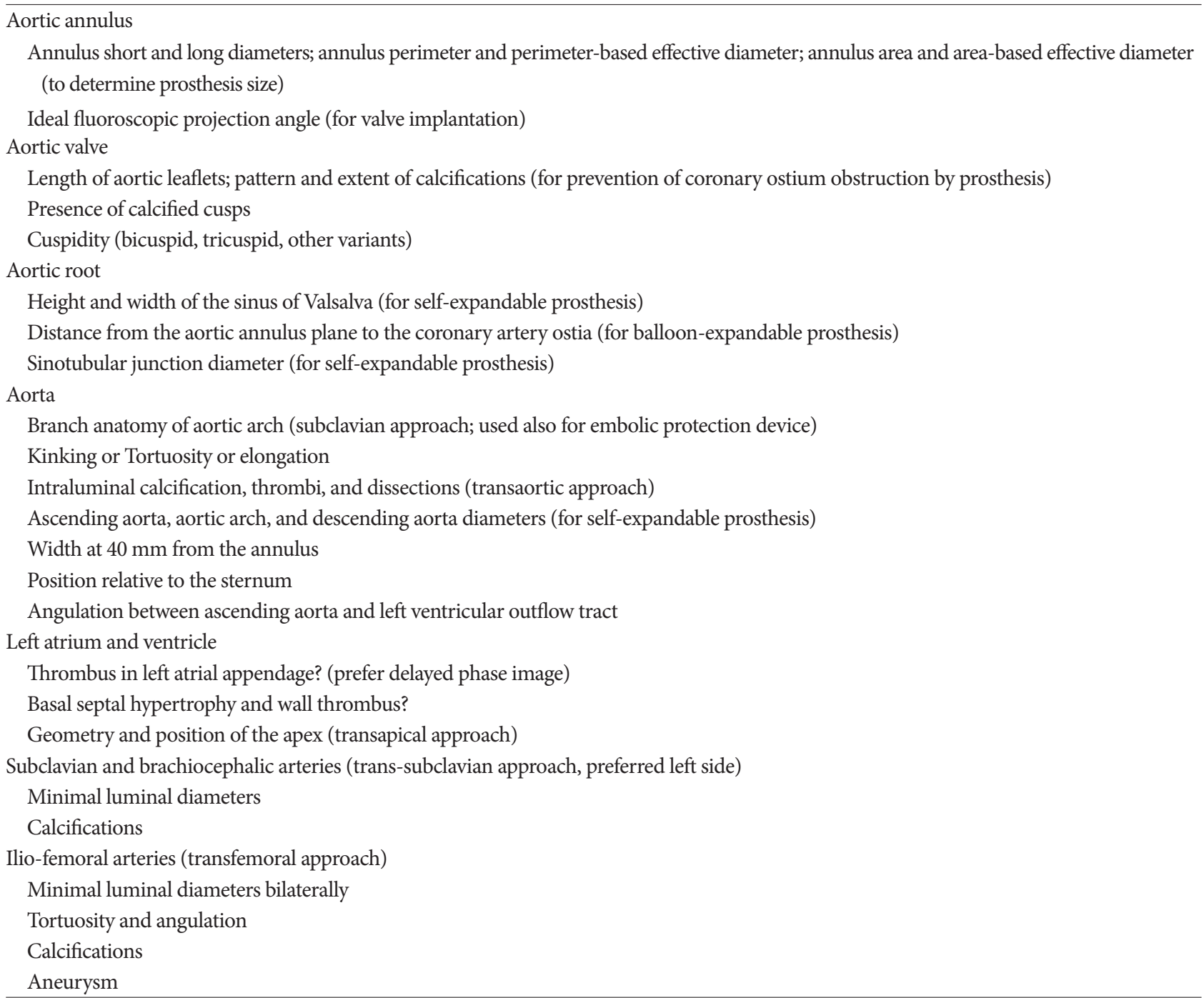

has been observed [35]. Although diffusion-weighted (DW) MRI has detected post-procedural ischemic brain lesions in more than two-thirds of patients, the clinical relevance of these brain lesions remains uncertain. One study that longitudinally followed patients with DW MRI-detected brain lesions reported complete resolution of the lesions after 6 months post-TAVR [36].

\section{Occlusion of the coronary ostia}

Coronary ostia may be occluded by either a malpositioned prosthetic device or displaced native valve leaflets, with a reported incidence rate of about $0.66 \%$ in one multicenter registry [37]. A close proximity of the coronary ostia to the valve annulus, bulky calcification of either the right or left valve leaflets, and a narrow sinus of Valsalva identified on MDCT imaging are the most significant predictors for this complication $[23,38]$. A low-lying coronary ostium $(<12 \mathrm{~mm})$ and a shallow sinus of Valsalva $(<30 \mathrm{~mm})$ are the two anatomic predictors most strong- ly associated with coronary obstruction $[37,38]$.

\section{Valve thrombosis}

All types of prostheses have thin leaflets $(<0.35 \mathrm{~mm})$, typically thinner than those of surgical valves, and normally appear as fine hypodense lines on MDCT. Studies have shown that thickening of one or more prosthetic valve leaflet on MDCT is indicative of valve thrombosis and is associated with a moderate increase in trans-prosthetic gradients. It is also speculated that this finding may be associated with an increased risk of thromboembolic events and reduced valve durability. With the use of MDCT for post-TAVR evaluation, subclinical prosthetic valve thrombosis has been increasingly recognized $[12,39,40]$.

\section{Injuries to the aorta and left ventricle}

Aortic dissection (Fig. 4), rupture of the aortic root, and injury to the left ventricle are rare but life-threating complications 

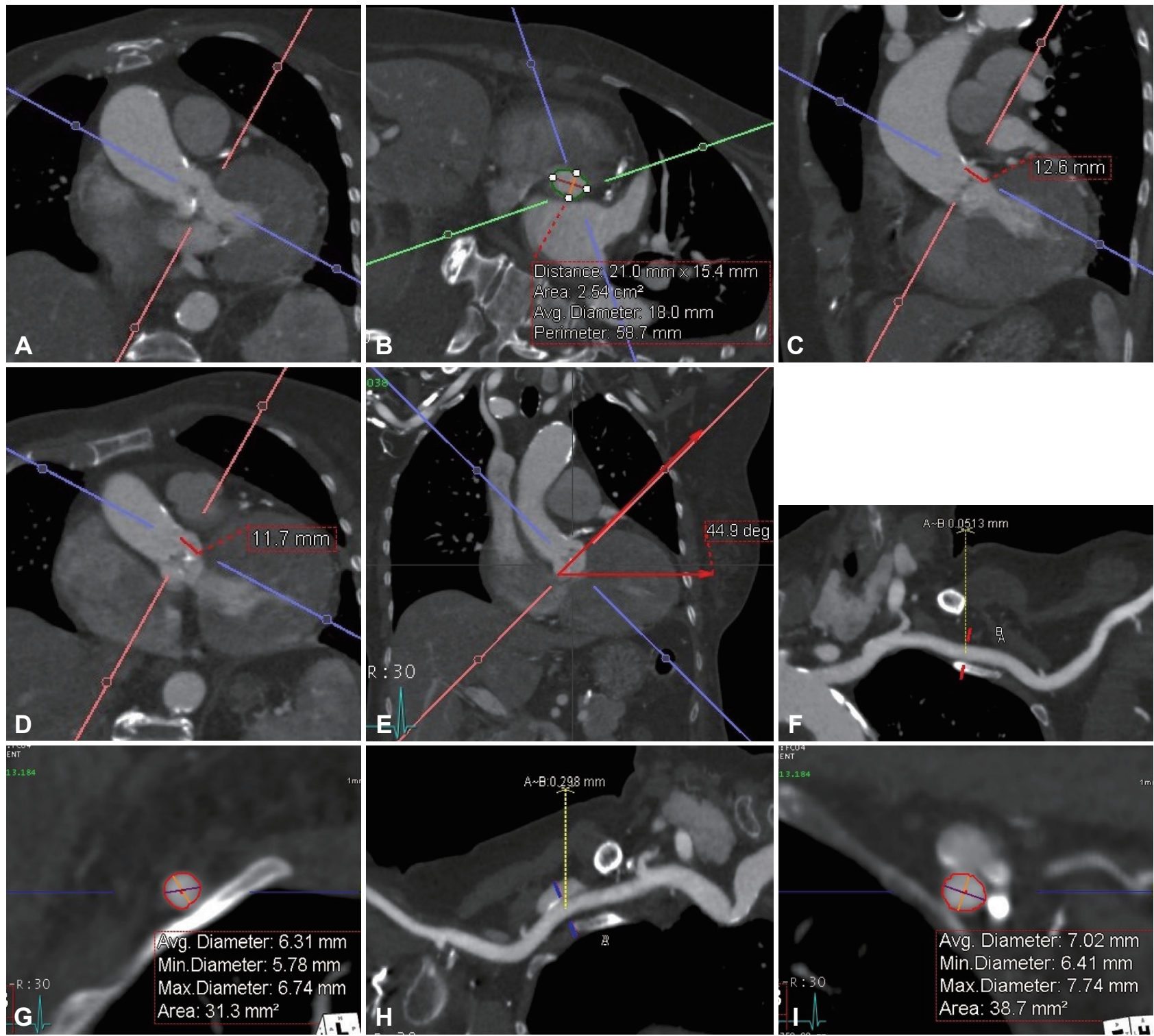

Fig. 3. Demonstration of multi-detector computed tomography measurements for pre-procedural transcatheter aortic valve replacement evaluation. (A) After sequential double-oblique reconstructions, all parameters including (B) maximal diameter, minimal diameter, average diameter and perimeter of the aortic annulus, (C) the distance from the annulus plane to the left coronary artery ostia, (D) the distance from the annulus plane to the right coronary artery ostia, $(E)$ the aortic root angle, $(F, G, H, I)$ the luminal diameter of bilateral subclavian arteries, $(J, K, L$, $\mathrm{M})$ the luminal diameter of bilateral common iliac arteries, and (N, O, P, Q) the luminal diameter of bilateral femoral arteries were measured.

of TAVR [41-43]. Aortic root rupture is more frequently encountered if balloon-expandable prosthetic devices are used in patients with calcified LVOT, particularly when the prostheses are aggressively oversized [44].

\section{Complications at the access routes}

Pseudoaneurysms, arteriovenous fistulas, and hematoma may occur at any of the peripheral vascular access sites for TAVR [33].

\section{POST-PROCEDURE EVALUATION}

Current guidelines recommend that patients who have under- gone TAVR should undergo transthoracic echocardiography before hospital discharge. The role of MDCT in the immediate post-procedure follow-up is limited. Echocardiography should be routinely performed within 30-90 days to establish baseline valve function, at 1 year after TAVR, and annually thereafter. Transvalvular gradient, the presence and the degree of PVR/central regurgitation, and the overall left ventricular function should be monitored during echocardiography (Fig. 5) [12].

Although its utility has not been fully established, MDCT angiography may be helpful in triaging patients who develop new onset of symptoms after a TAVR procedure. In addition, MDCT with multi-phase reconstruction and cine imaging offers evalu- 

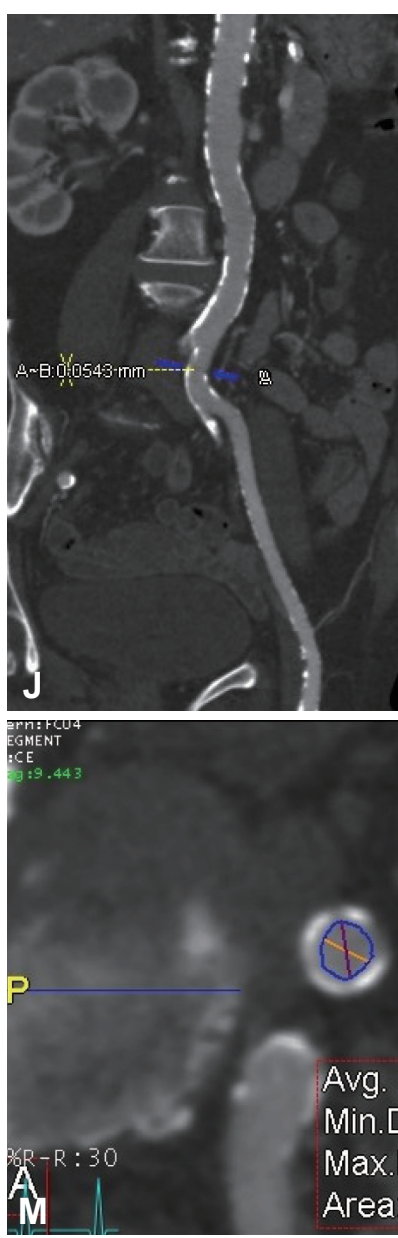

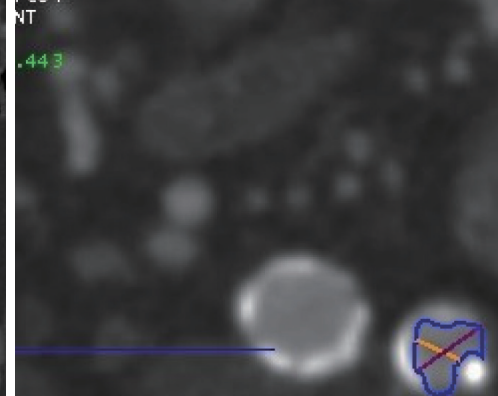

Avg. Diameter Min.Diameter: $5.00 \mathrm{~mm}$ Max.Diameter: $7.86 \mathrm{~mm}$ Area: $34.9 \mathrm{~mm}^{2}$

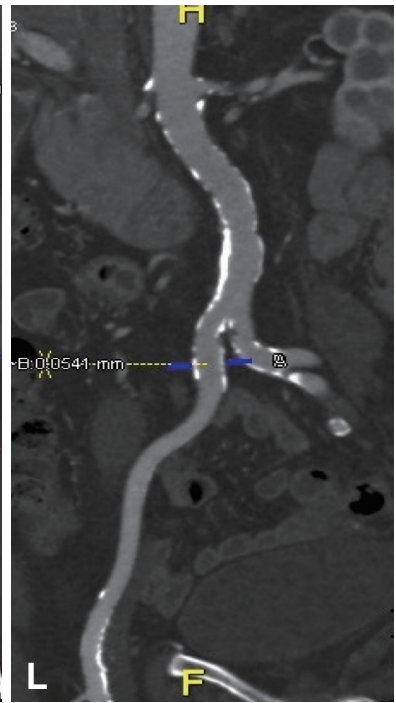

Avg. Diameter: $7.11 \mathrm{~mm}$ Min.Diameter: $6.66 \mathrm{~mm}$ Max.Diameter: $7.71 \mathrm{~mm}$ Area: $39.7 \mathrm{~mm}^{2}$
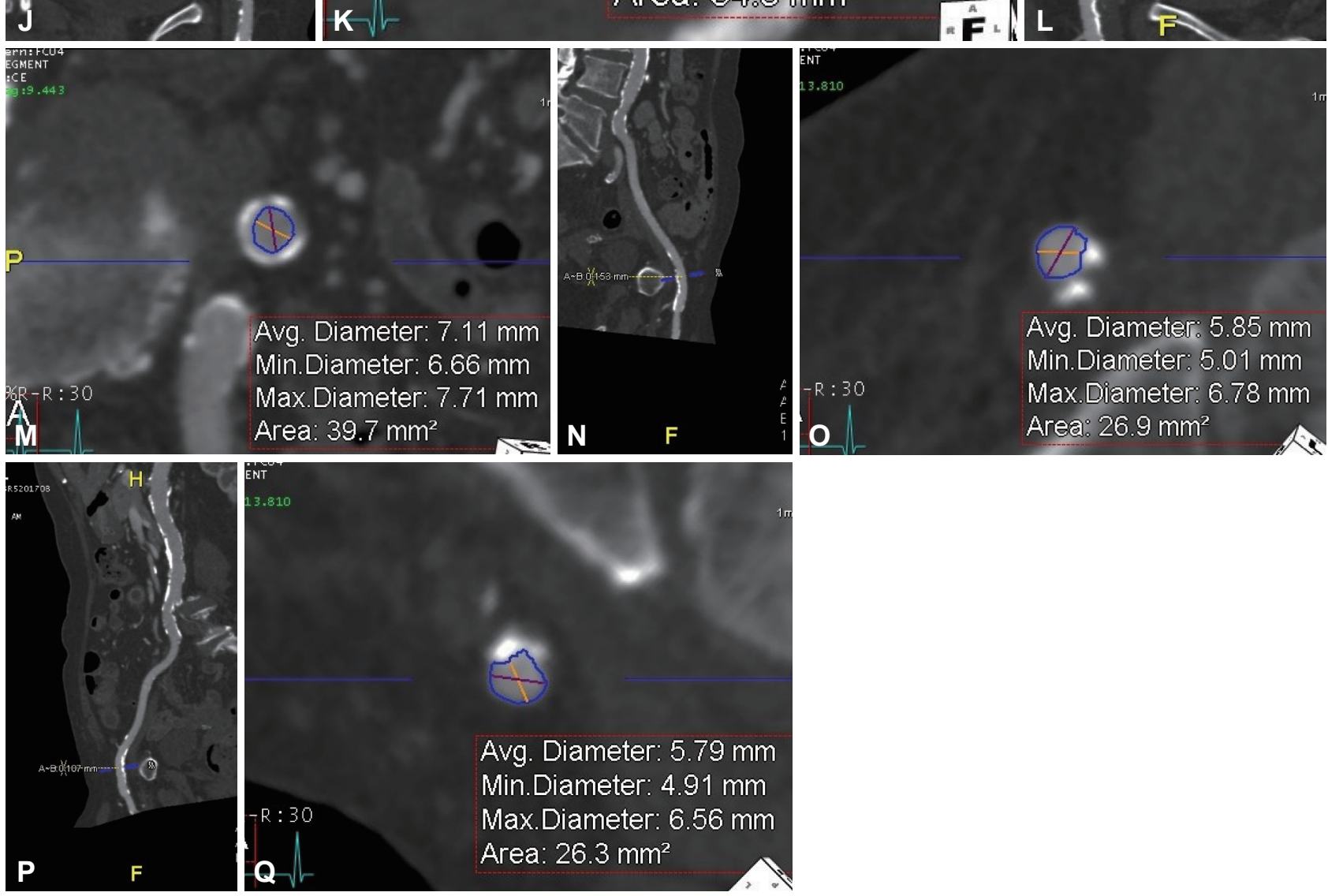

Fig. 3. Demonstration of multi-detector computed tomography measurements for pre-procedural transcatheter aortic valve replacement evaluation. (A) After sequential double-oblique reconstructions, all parameters including (B) maximal diameter, minimal diameter, average diameter and perimeter of the aortic annulus, (C) the distance from the annulus plane to the left coronary artery ostia, (D) the distance from the annulus plane to the right coronary artery ostia, $(\mathrm{E})$ the aortic root angle, $(\mathrm{F}, \mathrm{G}, \mathrm{H}, \mathrm{I})$ the luminal diameter of bilateral subclavian arteries, $(\mathrm{J}, \mathrm{K}, \mathrm{L}$, $\mathrm{M})$ the luminal diameter of bilateral common iliac arteries, and $(\mathrm{N}, \mathrm{O}, \mathrm{P}, \mathrm{Q})$ the luminal diameter of bilateral femoral arteries were measured.

ation of the morphology, position, expansion as well as the dynamic movement of the prosthesis valve leaflets [39]. Hypokinesia of a thickened prosthetic leaflet usually suggests dysfunction of the prosthetic device. The post-TAVR MDCT protocol we recommended is summarized in Table 5 [33].

\section{SPECIAL ISSUES IN THE ASIAN POPULATION}

The number of candidates for TAVR is expected to rise in Asia, especially in the North Eastern region of Asia where elderly populations are growing. Some anatomical features specific to 

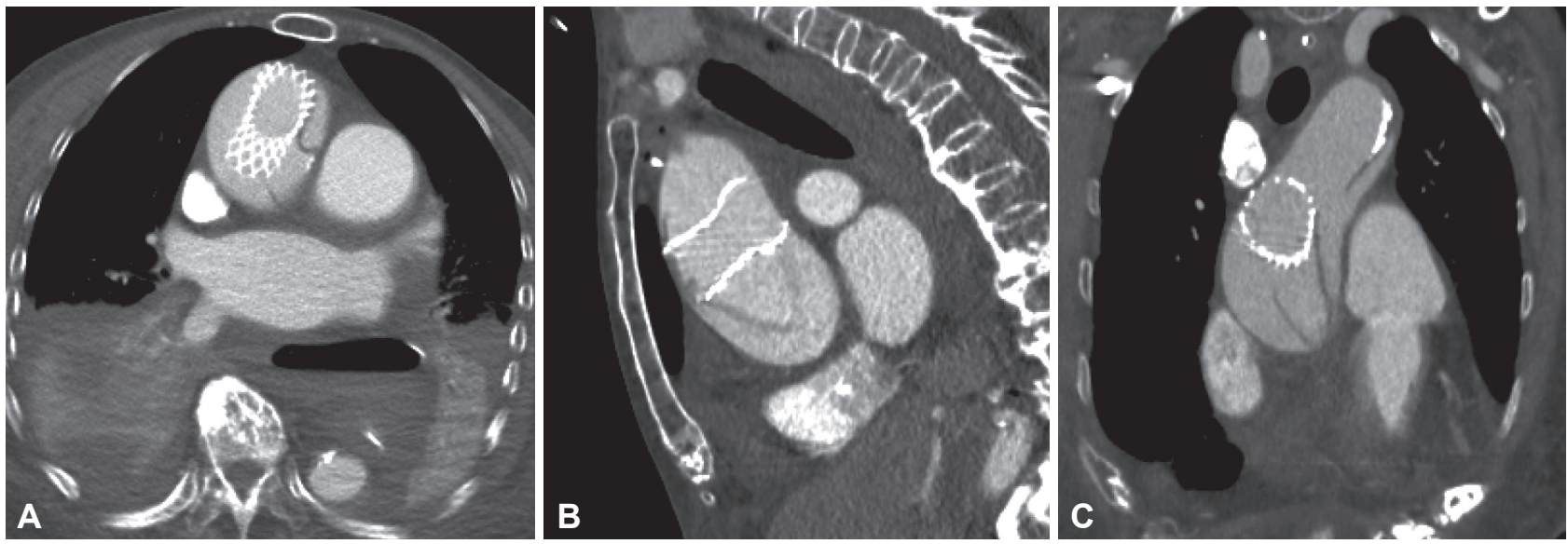

Fig. 4. A 91-year-old female with severe aortic valve stenosis underwent TAVR (Medtronic Evolut $R 29 \mathrm{~mm}$ valve). Post-TAVR multi-detector computed tomography in (A) axial $(B)$ sagittal and $(C)$ coronal views showed concomitant migration of the prosthetic device into the ascending aorta and type A aortic dissection, which progressed to cardiac temponade and led to her death within one day after the TAVR. TAVR: transcatheter aortic valve replacement.
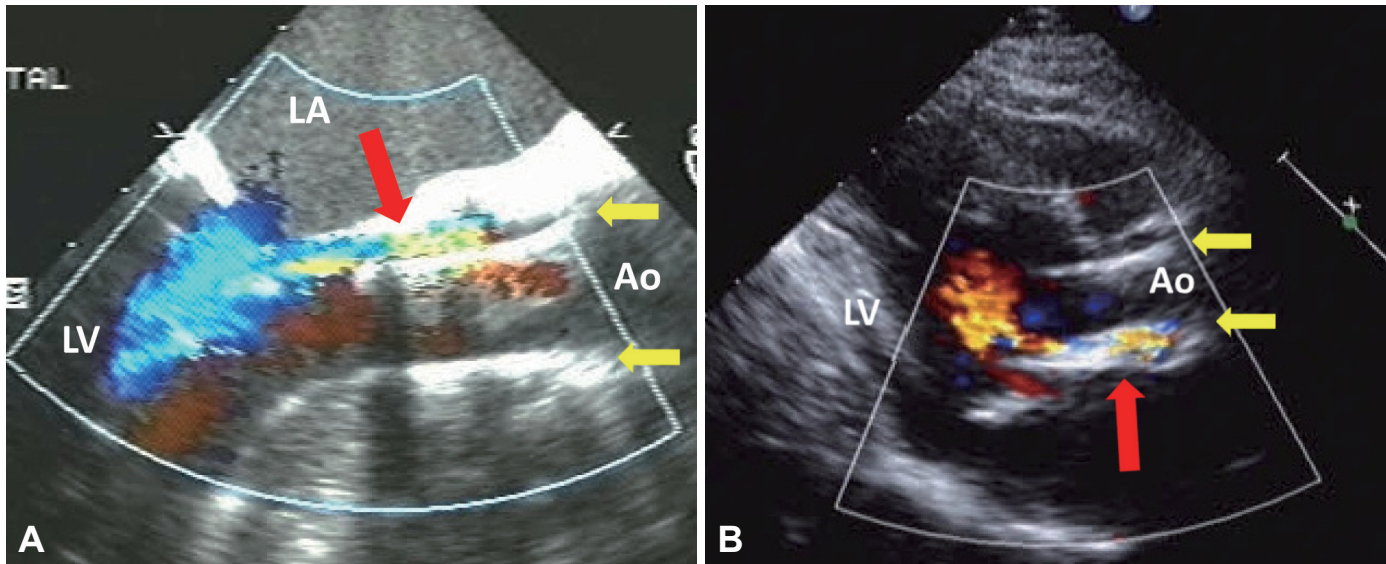

Fig. 5. An 81-year-old female with severe aortic valve stenosis being treated with TAVR (Medtronic CoreValve). Both immediate post-TAVR transesophageal echocardiography in long axial view (A) and transthoracic echocardiography 30 days after TAVR (B) revealed color jets (red arrow) running in between the prosthetic device (yellow arrows) and the aortic wall, indicating moderate paravalvular regurgitation. Ao: aorta, LA: left atrium, LV: left ventricle, TAVR: transcatheter aortic valve replacement.

Asian patients may cause more complications and lead to increased mortality. The more prevalent small annulus and lower left coronary height in an Asian cohort is associated with a higher incidence of coronary obstruction (approximately 1.3\%). In addition, the prevalence of bicuspid aortic valve (BAV) has been reported to be greater than $5.8 \%$ in Asians, which is higher than rates reported in Europe and North America (1.3\% to 4.7\%) [45-47].

\section{MINIMALIZED INVASIVENESS OF TAVR}

The core strength of TAVR lies in its minimal invasiveness. The advantages of TAVR over SAVR include decreased procedural time, lower complication rates, shortened hospital length of stay, and reduced cost. However, studies on how to safely and efficaciously perform the procedure without the need for general anesthesia or TEE guidance are still ongoing [20,48-50].

\section{BICUSPID AORTIC VALVES}

BAV-a common congenital anomaly-is susceptible to mechanical shear stress, which over time causes fibrosis, calcification, and stenosis of the valve (Fig. 6). The phenotypes of BAV are classified based on the presence or absence of raphe (commissural fusion) and the number of raphe if present. In Sievers' classification, BAV is described as follows: type 0 , without raphe; type 1 , with one raphe; and type 2 , with two raphes. BAV Imaging classification categorizes BAV into three types: tricommissural, bicommissural raphe, and bicommissural non-raphe [51,52]. In two Asian multicenter studies more than $95 \%$ of BAV was documented as the bicommissural type, $62-93 \%$ of which were the non-raphe subtype $[51,53]$. Evidence has shown that due to the abnormal geometry and eccentricity of the native bicuspid valve and the often co-existing dilatation of the ascending aorta, patients with BAV receiving TAVR may be predisposed to 
Table 5. Post-procedural transcatheter aortic valve replacement multi-detector computed tomography protocol recommendations

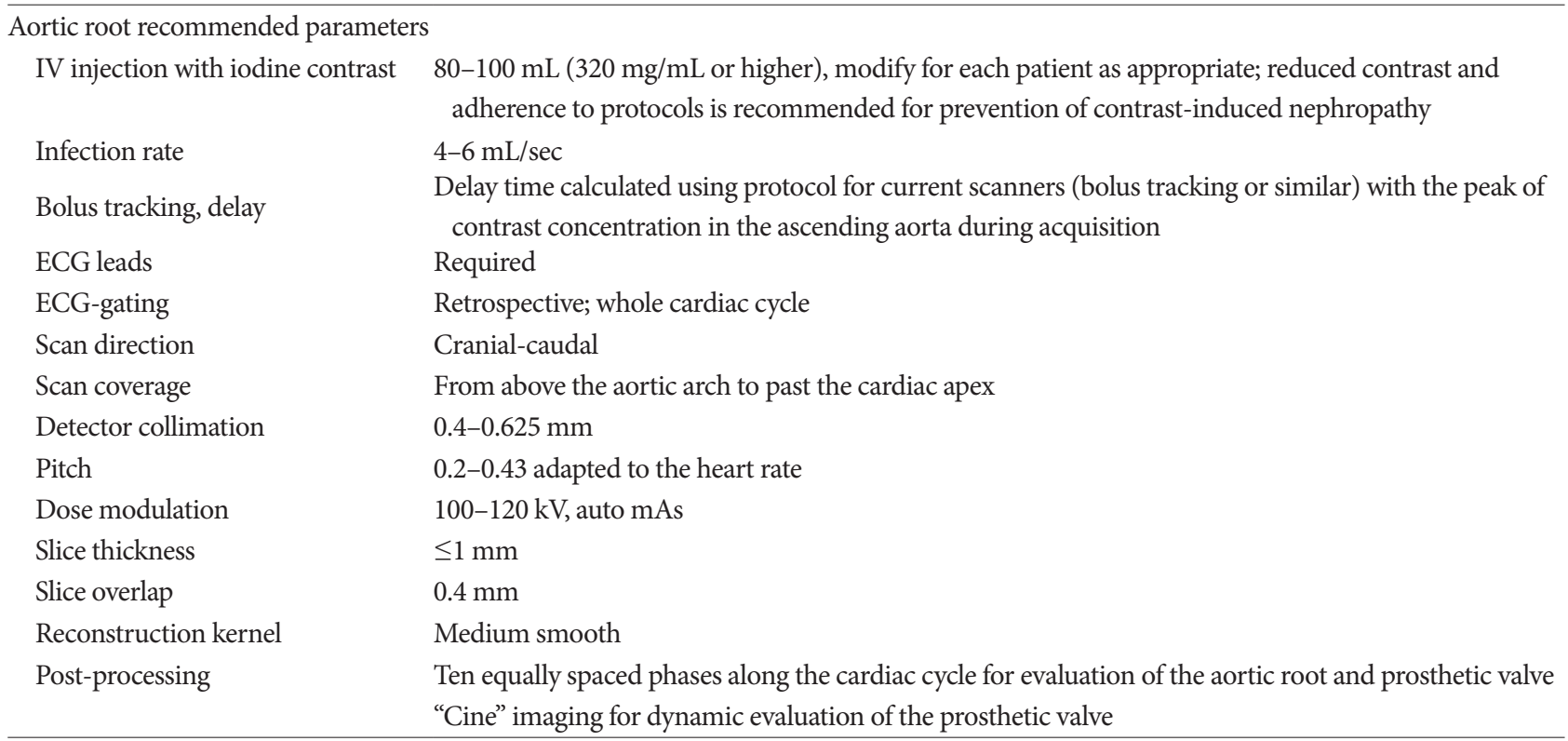

ECG: electrocardiography
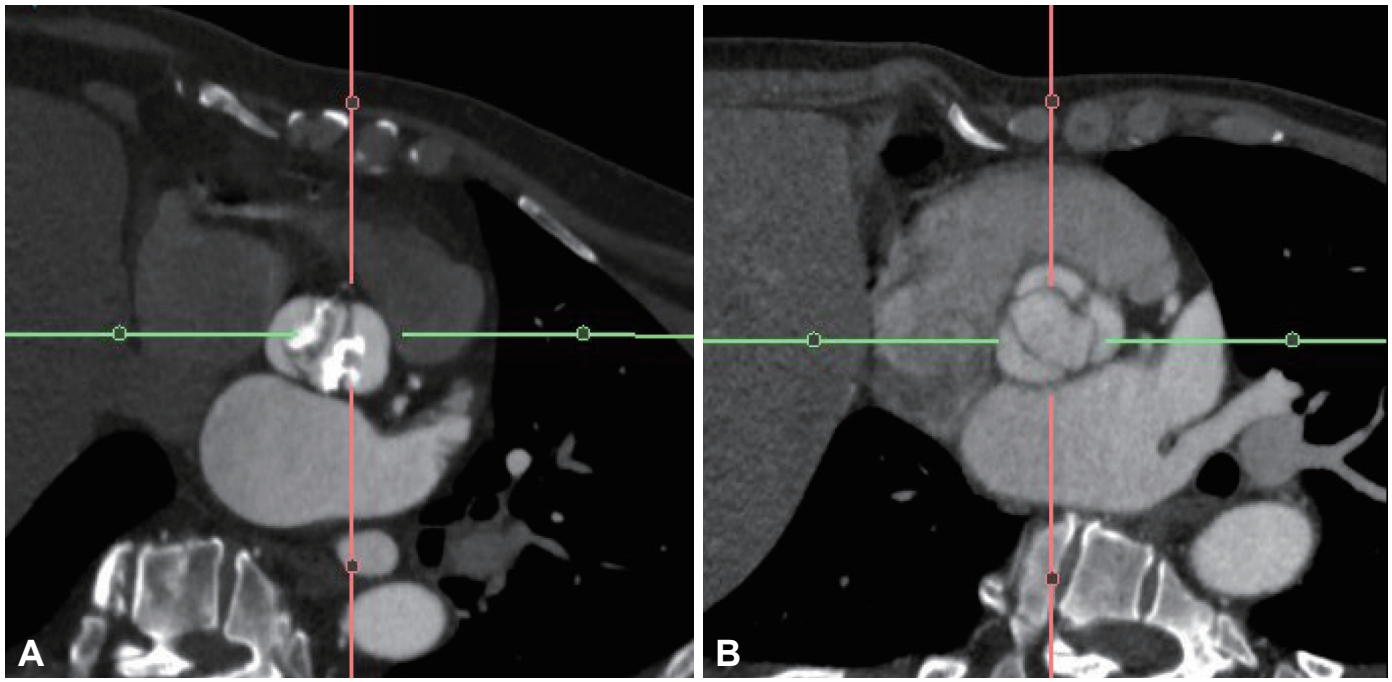

Fig. 6. (A) A 63-year-old male with severe AS. Transverse MDCT image showed a Sievers classification type 0 (without raphe) BAV with prominent leaflet calcification. Surgical aortic valve replacement was performed due to his heavily calcified BAV. (B) A 59-year-old male with mild AS. Transverse MDCT image showed a Sievers classification type 1 (raphe and two commissures) BAV. He was medically treated and advised to follow up. AS: aortic valve stenosis, MDCT: multi-detector computed tomography, BAV: bicuspid aortic valve.

mal-apposition, inadequate anchoring, and improper positioning of the prosthesis, resulting in significant PVR and prosthetic malfunction [20,54,55]. Although current guidelines advise against TAVR as the first-line treatment for severe AS in the setting of congenital BAV, more recent evidence from observational studies has shown that TAVR for bicuspid AS patients yielded a low 30-day mortality rate and, perhaps more importantly, a high success rate comparable to that of TAVR for tricuspid AS patients, especially if newer generations of prosthetic devices are used. Nevertheless, a higher complication rate of significant PVR and PPI in the BAV group was still observed [56-58]. More stud- ies on BAV with respect to the methodology of annular sizing, valvular shape and function, the durability of prosthesis devices, and concomitant aortopathy in BAV patients, in addition to outcome studies and randomized controlled trials particularly targeting younger BAV patients, are required before drawing final conclusion $[59,60]$.

\section{CONCLUSION}

TAVR still faces some challenges. The suboptimal results of TAVR in patients with BAV require further study. Issues regard- 
ing the feasibility of TAVR in younger patient groups, the longterm durability of the prosthetic devices, the introduction of a more personalized prosthesis device catering to individual needs, and many more issues should be addressed in the future.

MDCT is the recommended image modality for pre-procedural planning of the TAVR procedure. TAVR MDCT should be performed by well-trained personnel and interpreted and analyzed by experienced radiologists who not only have a sound understanding of TAVR with respect to its indications, prosthetic devices, procedure, and complications but also work in close collaboration with the Heart Team.

\section{Conflicts of Interest}

The authors declare that they have no conflict of interest.

\section{Acknowledgments}

The authors would like to thank Dr. Yi-Chang Chen for providing the rare and valuable case of post-TAVR complication.

\section{REFERENCES}

1. Coffey S, Cox B, Williams MJ. The prevalence, incidence, progression, and risks of aortic valve sclerosis: a systematic review and meta-analysis. J Am Coll Cardiol 2014;63:2852-2861.

2. Otto CM, Prendergast B. Aortic-valve stenosis--from patients at risk to severe valve obstruction. N Engl J Med 2014;371:744-756.

3. Varadarajan P, Kapoor N, Bansal RC, Pai RG. Survival in elderly patients with severe aortic stenosis is dramatically improved by aortic valve replacement: results from a cohort of 277 patients aged $>$ or $=80$ years. Eur J Cardiothorac Surg 2006;30:722-727.

4. Vasques F, Messori A, Lucenteforte E, Biancari F. Immediate and late outcome of patients aged 80 years and older undergoing isolated aortic valve replacement: a systematic review and meta-analysis of 48 studies. Am Heart J 2012;163:477-485.

5. Smith CR, Leon MB, Mack MJ, Miller DC, Moses JW, Svensson LG, et al. Transcatheter versus surgical aortic-valve replacement in high-risk patients. N Engl J Med 2011;364:2187-2198.

6. Adams DH, Popma JJ, Reardon MJ, Yakubov SJ, Coselli JS, Deeb GM, et al. Transcatheter aortic-valve replacement with a self-expanding prosthesis. N Engl J Med 2014;370:1790-1798.

7. Thyregod HG, Steinbrüchel DA, Ihlemann N, Nissen H, Kjeldsen BJ, Petursson $\mathrm{P}$, et al. Transcatheter versus surgical aortic valve replacement in patients with severe aortic valve stenosis: 1-year results from the all-comers NOTION randomized clinical trial. J Am Coll Cardiol 2015;65:21842194.

8. Leon MB, Smith CR, Mack MJ, Makkar RR, Svensson LG, Kodali SK, et al. Transcatheter or surgical aortic-valve replacement in intermediaterisk patients. N Engl J Med 2016;374:1609-1620.

9. Rosato S, Santini F, Barbanti M, Biancari F, D’Errigo P, Onorati F, et al. Transcatheter aortic valve implantation compared with surgical aortic valve replacement in low-risk patients. Circ Cardiovasc Interv 2016;9: e003326.

10. Arora S, Strassle PD, Ramm CJ, Rhodes JA, Vaidya SR, Caranasos TG, et al. Transcatheter versus surgical aortic valve replacement in patients with lower surgical risk scores: a systematic review and meta-analysis of early outcomes. Heart Lung Circ 2017;26:840-845.

11. Reardon MJ, Van Mieghem NM, Popma JJ, Kleiman NS, Søndergaard L, Mumtaz M, et al. Surgical or transcatheter aortic-valve replacement in intermediate-risk patients. N Engl J Med 2017;376:1321-1331.

12. Thonghong T, De Backer O, Søndergaard L. Comprehensive update on the new indications for transcatheter aortic valve replacement in the lat- est 2017 European guidelines for the management of valvular heart disease. Open Heart 2018;5:e000753.

13. Baumgartner H, Falk V, Bax JJ, De Bonis M, Hamm C, Holm PJ, et al. 2017 ESC/EACTS Guidelines for the management of valvular heart disease. Eur Heart J 2017;38:2739-2791.

14. Nishimura RA, Otto CM, Bonow RO, Carabello BA, Erwin JP 3rd, Fleisher LA, et al. 2017 AHA/ACC focused update of the 2014 AHA/ACC guideline for the management of patients with valvular heart disease: a report of the american college of cardiology/american heart association task force on clinical practice guidelines. Circulation 2017;135:e1159-e1195.

15. Otto CM, Kumbhani DJ, Alexander KP, Calhoon JH, Desai MY, Kaul S, et al. 2017 ACC expert consensus decision pathway for transcatheter aortic valve replacement in the management of adults with aortic stenosis: a report of the american college of cardiology task force on clinical expert consensus documents. J Am Coll Cardiol 2017;69:1313-1346.

16. Baumgartner H. The 2017 ESC/EACTS guidelines on the management of valvular heart disease: what is new and what has changed compared to the 2012 guidelines? Wien Klin Wochenschr 2018;130:168-171.

17. Shahian DM, Jacobs JP, Badhwar V, Kurlansky PA, Furnary AP, Cleveland JC Jr, et al. The society of thoracic surgeons 2018 adult cardiac surgery risk models: part 1-background, design considerations, and model development. Ann Thorac Surg 2018;105:1411-1418.

18. O’Brien SM, Feng L, He X, Xian Y, Jacobs JP, Badhwar V, et al. The society of thoracic surgeons 2018 adult cardiac surgery risk models: part 2-statistical methods and results. Ann Thorac Surg 2018;105:1419-1428.

19. Nashef SA, Roques F, Sharples LD, Nilsson J, Smith C, Goldstone AR, et al. EuroSCORE II. Eur J Cardiothorac Surg 2012;41:734-744 discussion 744-745.

20. Young MN, Inglessis I. Transcatheter aortic valve replacement: outcomes, indications, complications, and innovations. Curr Treat Options Cardiovasc Med 2017;19:81.

21. Chamandi C, Puri R, Rodriguez-Gabella T, Rodés-Cabau J. Latest-generation transcatheter aortic valve replacement devices and procedures. Can J Cardiol 2017;33:1082-1090.

22. Agarwal S, Parashar A, Kumbhani DJ, Svensson LG, Krishnaswamy A, Tuzcu EM, et al. Comparative meta-analysis of balloon-expandable and self-expandable valves for transcatheter aortic valve replacement. Int J Cardiol 2015;197:87-97.

23. Ali N, Patel PA, Lindsay SJ. Recent developments and controversies in transcatheter aortic valve implantation. Eur J Heart Fail 2018;20:642-650.

24. Achenbach S, Delgado V, Hausleiter J, Schoenhagen P, Min JK, Leipsic JA. SCCT expert consensus document on computed tomography imaging before transcatheter aortic valve implantation (TAVI)/transcatheter aortic valve replacement (TAVR). J Cardiovasc Comput Tomogr 2012;6:366380.

25. Salgado RA, Leipsic JA, Shivalkar B, Ardies L, Van Herck PL, Op de Beeck BJ, et al. Preprocedural CT evaluation of transcatheter aortic valve replacement: what the radiologist needs to know. Radiographics 2014; 34:1491-1514.

26. Wuest W, Anders K, Schuhbaeck A, May MS, Gauss S, Marwan M, et al. Dual source multidetector CT-angiography before Transcatheter Aortic Valve Implantation (TAVI) using a high-pitch spiral acquisition mode. Eur Radiol 2012;22:51-58.

27. Geyer LL, De Cecco CN, Schoepf UJ, Silverman JR, Krazinski AW, Bamberg F, et al. Low-volume contrast medium protocol for comprehensive cardiac and aortoiliac CT assessment in the context of transcatheter aortic valve replacement. Acad Radiol 2015;22:1138-1146.

28. Pulerwitz TC, Khalique OK, Nazif TN, Rozenshtein A, Pearson GD, Hahn $\mathrm{RT}$, et al. Very low intravenous contrast volume protocol for computed tomography angiography providing comprehensive cardiac and vascular assessment prior to transcatheter aortic valve replacement in patients with chronic kidney disease. J Cardiovasc Comput Tomogr 2016;10:316-321.

29. Leipsic J, Gurvitch R, Labounty TM, Min JK, Wood D, Johnson M, et al. Multidetector computed tomography in transcatheter aortic valve implantation. JACC Cardiovasc Imaging 2011;4:416-429. 
30. Latsios G, Spyridopoulos TN, Toutouzas K, Synetos A, Trantalis G, Stathogiannis K, et al. Multi-slice CT (MSCT) imaging in pretrans-catheter aortic valve implantation (TAVI) screening. How to perform and how to interpret. Hellenic J Cardiol 2018;59:3-7.

31. Pascual I, Carro A, Avanzas P, Hernández-Vaquero D, Díaz R, Rozado J, et al. Vascular approaches for transcatheter aortic valve implantation. J Thorac Dis 2017;9(Suppl 6):S478-S487.

32. Toggweiler S, Gurvitch R, Leipsic J, Wood DA, Willson AB, Binder RK, et al. Percutaneous aortic valve replacement: vascular outcomes with a fully percutaneous procedure. J Am Coll Cardiol 2012;59:113-118.

33. Salgado RA, Budde RP, Leiner T, Shivalkar B, Van Herck PL, Op de Beeck BJ, et al. Transcatheter aortic valve replacement: postoperative CT findings of Sapien and CoreValve transcatheter heart valves. Radiographics 2014;34:1517-1536.

34. Takagi H, Umemoto T; ALICE (All-Literature Investigation of Cardiovascular Evidence) Group. Impact of paravalvular aortic regurgitation after transcatheter aortic valve implantation on survival. Int J Cardiol 2016;221:46-51.

35. Athappan G, Gajulapalli RD, Sengodan P, Bhardwaj A, Ellis SG, Svensson $\mathrm{L}$, et al. Influence of transcatheter aortic valve replacement strategy and valve design on stroke after transcatheter aortic valve replacement: a metaanalysis and systematic review of literature. J Am Coll Cardiol 2014;63: 2101-2110.

36. Lansky AJ, Schofer J, Tchetche D, Stella P, Pietras CG, Parise H, et al. A prospective randomized evaluation of the TriGuard HDH embolic DEFLECTion device during transcatheter aortic valve implantation: results from the DEFLECT III trial. Eur Heart J 2015;36:2070-2078.

37. Ribeiro HB, Webb JG, Makkar RR, Cohen MG, Kapadia SR, Kodali S, et al. Predictive factors, management, and clinical outcomes of coronary obstruction following transcatheter aortic valve implantation: insights from a large multicenter registry. J Am Coll Cardiol 2013;62:1552-1562.

38. Barbanti M. Avoiding coronary occlusion and root rupture in TAVI - the role of pre-procedural imaging and prosthesis selection. Interv Cardiol 2015;10:94-97.

39. Soschynski M, Capilli F, Ruile P, Neumann FJ, Langer M, Krauss T. PostTAVI follow-up with MDCT of the valve prosthesis: technical application, regular findings and typical local post-interventional complications. Rofo 2018;190:521-530.

40. Chakravarty T, Søndergaard L, Friedman J, De Backer O, Berman D, Kofoed KF, et al. Subclinical leaflet thrombosis in surgical and transcatheter bioprosthetic aortic valves: an observational study. Lancet 2017;389:23832392.

41. Langer NB, Hamid NB, Nazif TM, Khalique OK, Vahl TP, White J, et al. Injuries to the aorta, aortic annulus, and left ventricle during transcatheter aortic valve replacement: management and outcomes. Circ Cardiovasc Interv 2017;10. e004735.

42. Kakefuda Y, Hayashida K, Yamada Y, Yashima F, Inohara T, Yanagisawa R, et al. Impact of subclinical vascular complications detected by systematic postprocedural multidetector computed tomography after transcatheter aortic valve implantation using balloon-expandable edwards SAPIEN XT heart valve. Am J Cardiol 2017;119:1100-1105.

43. Walther T, Hamm CW, Schuler G, Berkowitsch A, Kötting J, Mangner N, et al. Perioperative results and complications in 15,964 transcatheter aortic valve replacements: prospective data from the GARY registry. J Am Coll Cardiol 2015;65:2173-2180.

44. Möllmann H, Kim WK, Kempfert J, Walther T, Hamm C. Complications of transcatheter aortic valve implantation (TAVI): how to avoid and treat them. Heart 2015;101:900-908.

45. Costopoulos C, Latib A, Maisano F, Testa L, Bedogni F, Buchanan L, et al. Comparison of results of transcatheter aortic valve implantation in patients with severely stenotic bicuspid versus tricuspid or nonbicuspid valves. Am J Cardiol 2014;113:1390-1393.

46. Yoon SH, Ohno Y, Araki M, Barbanti M, Lin MS, Ahn JM, et al. Comparison of aortic root anatomy and calcification distribution between asian and caucasian patients who underwent transcatheter aortic valve implantation. Am J Cardiol 2015;116:1566-1573.

47. Yoon SH, Ahn JM, Hayashida K, Watanabe Y, Shirai S, Kao HL, et al. Clinical outcomes following transcatheter aortic valve replacement in asian population. JACC Cardiovasc Interv 2016;9:926-933.

48. Wiegerinck EM, Boerlage-van Dijk K, Koch KT, Yong ZY, Vis MM, Planken RN, et al. Towards minimally invasiveness: transcatheter aortic valve implantation under local analgesia exclusively. Int J Cardiol 2014;176: 1050-1052.

49. Attizzani GF, Ohno Y, Latib A, Petronio AS, De Carlo M, Giannini C, et al. Transcatheter aortic valve implantation under angiographic guidance with and without adjunctive transesophageal echocardiography. Am J Cardiol 2015;116:604-611.

50. Jensen HA, Condado JF, Devireddy C, Binongo J, Leshnower BG, Babaliaros V, et al. Minimalist transcatheter aortic valve replacement: The new standard for surgeons and cardiologists using transfemoral access? J Thorac Cardiovasc Surg 2015;150:833-839.

51. Jilaihawi $\mathrm{H}$, Chen M, Webb J, Himbert D, Ruiz CE, Rodés-Cabau J, et al. A bicuspid aortic valve imaging classification for the TAVR Era. JACC Cardiovasc Imaging 2016;9:1145-1158.

52. Sievers HH, Schmidtke C. A classification system for the bicuspid aortic valve from 304 surgical specimens. J Thorac Cardiovasc Surg 2007;133: 1226-1233.

53. Kong WKF, Regeer MV, Poh KK, Yip JW, van Rosendael PJ, Yeo TC, et al. Inter-ethnic differences in valve morphology, valvular dysfunction, and aortopathy between Asian and European patients with bicuspid aortic valve. Eur Heart J 2018;39:1308-1313.

54. Praz F, Windecker S, Huber C, Carrel T, Wenaweser P. Expanding indications of transcatheter heart valve interventions. JACC Cardiovasc Interv 2015;8:1777-1796.

55. Tousoulis $\mathrm{D}$. The evolution in the management of aortic valve disease: From surgical techniques to transcatheter interventions. Hellenic J Cardiol 2016;57:379-381.

56. Reddy G, Wang Z, Nishimura RA, Greason KL, Yoon SH, Makkar RR, et al. Transcatheter aortic valve replacement for stenotic bicuspid aortic valves: Systematic review and meta analyses of observational studies. Catheter Cardiovasc Interv 2018;91:975-983.

57. Yoon SH, Sharma R, Chakravarty T, Kawamori H, Maeno Y, Miyasaka $\mathrm{M}$, et al. Clinical outcomes and prognostic factors of transcatheter aortic valve implantation in bicuspid aortic valve patients. Ann Cardiothorac Surg 2017;6:463-472.

58. Yoon SH, Bleiziffer S, De Backer O, Delgado V, Arai T, Ziegelmueller J, et al. Outcomes in transcatheter aortic valve replacement for bicuspid versus tricuspid aortic valve stenosis. J Am Coll Cardiol 2017;69:2579-2589.

59. Yoon SH, Sharma R, Chakravarty T, Miyasaka M, Ochiai T, Nomura T, et al. Transcatheter aortic valve replacement in bicuspid aortic valve stenosis: where do we stand? J Cardiovasc Surg (Torino) 2018;59:381-391.

60. De Backer O, Søndergaard L. TAVR in bicuspid aortic valve stenosis: "We are not there yet" to draw final conclusions. Catheter Cardiovasc Interv 2018;91:984-985. 